\title{
Escala de Machismo Sexual (EMS-Sexismo-12): diseño y análisis de propiedades psicométricas
}

\section{Design and psychometric properties of The Sexual Machism Scale (EMS-Sexism-12)}

\author{
Cecilia L. Díaz Rodríguez ${ }^{1}$, María A. Rosas Rodríguez \& \\ Mónica Teresa González Ramírez \\ Universidad Autónoma de Nuevo León
}

(Recepción: Septiembre 2010 - Aceptación: Noviembre 2010)

\begin{abstract}
Resumen
La Escala de Machismo Sexual (Sexismo) (EMS-Sexismo-12) fue elaborada por las autoras del presente con la finalidad de evaluar, en hombres y mujeres, el nivel de machismo/sexismo que presentan, lo cual consideramos que puede derivar en conductas de riesgo en salud sexual. Actualmente no existe una escala en relación al Machismo Sexual. La escala se analizó con datos de 79 participantes, 43 hombres y 36 mujeres, con edades que van desde los 11 hasta 76 años. Originalmente se elaboraron 24 ítems, los análisis factoriales exploratorio y confirmatorio guiaron la reducción de la escala. Se logra obtener una escala breve y precisa (12 items, alfa de 0.91) y un solo factor que explica el $98.1 \%$ de varianza en el AFC, el cual presenta adecuados estadísticos de bondad de ajuste. Finalmente, al realizar la comparación entre hombres y mujeres se encontró una diferencia significativa siendo los hombres quienes presentan puntajes más altos. Palabras claves: Machismo, sexismo, infecciones de transmisión sexual, VIH/SIDA.
\end{abstract}

\begin{abstract}
The Sexual Machism Scale (EMS-Sexismo-12) evaluates in both men and women, sexism levels that could result in sexual health risk behaviors. Nowadays there is no Sexual Machism Scale. The analyses were made with 79 participants, 43 men and 36 women, with ages that go from 11 to 76 years. Originally 24 items were elaborated, however exploratory and confirmatory factor analysis guided the reduction of the scale. It is achieved to obtain a brief and precise scale (12 items, 0.91 alpha) with only one factor that explains $98.1 \%$ of its variance and adequate goodness of fit indices in confirmatory factor analysis. We also analyzed differences between men and women founding higher scores in men than in women.
\end{abstract}

Key words: Machism, sexism, sexually transmitted disease, HIV/AIDS.

\section{Introducción}

El machismo se puede definir como un conjunto de creencias, actitudes y conductas que manifiestan la superioridad del hombre sobre la mujer en áreas consideradas importantes para los hombres (Castañeda, 2002, 2007). Sin embargo la visión del machismo en la actualidad no es tan radical como en años anteriores. El hombre machista de ahora no golpea indiscriminadamente a la mujer

1 Correspondencia dirigida a: Cecilia L. Díaz Rodríguez. Av. Dr. Carlos Canseco \#110. Col. Mitras Centro CP 64460. Monterrey, N.L. México. Tel.: 01 (81) 83-48-38-66 y 01 (81) 83-48-02-86. E-mail: cecydiaz_@hotmail.com. 
como lo hacía antes, no obliga a su pareja a tener relaciones sexuales sin recibir un acto de defensa por parte de ella ni prohíbe a sus hijas mujeres estudiar una carrera; sin embargo, el machismo al que nos enfrentamos ahora es un machismo encubierto, donde se ejerce presión psicológica en contra de la mujer a quien se considera inferior, es luchar en contra de la mujer, demeritarla y tratar de dominar y humillar a la pareja sentimental.

Bonino (en Villegas, 2004) ha identificado una serie de comportamientos machistas que los hombres llevan a cabo de manera oculta e invisible los cuales ha denominado "micromachismos". Los micromachismos no se refieren a las formas extremas y notorias de violencia, más bien se refiere a aquellas conductas sutiles o suaves que son formas de violencia y abuso cotidiano. Son casi imperceptibles, ya que se trata de aquellos comportamientos relacionados con la educación social que se les da a los hombres y los ubica en una posición de ventaja con respecto a la mujer. El principal objetivo de los micromachismos es garantizar el control sobre la mujer. Todos los tipos de micromachismo buscan disminuir la libertad de elegir y decidir de las mujeres.

En la búsqueda de información se han encontrado investigaciones que hacen referencia al tema del machismo y lo denominan sexismo, para la presente investigación serán utilizados ambos términos indiscriminadamente. El sexismo puede definirse como una actitud de prejuicio o conducta discriminatoria basada en la supuesta inferioridad o diferencia de las mujeres como grupo (Cameron en Moya \& Expósito, 2001).

La teoría del sexismo ambivalente propuesta por Glick y Fiske (1996) pone de manifiesto que en este tipo de sexismo coexisten actitudes tanto positivas como negativas hacia las mujeres; las actitudes negativas las denominan sexismo hostil, el cuál es la manifestación clásica y tradicional del sexismo, y las actitudes positivas las denominan sexismo benevolente, en donde se justifican las actitudes de protección hacia las mujeres. Los autores ponen de manifiesto que ambos tipos de sexismo (hostil y benévolo) pueden coexistir y no son mutuamente excluyentes, de acuerdo a los autores la conceptualización tradicional del prejuicio como hostilidad hacia las mujeres, deja a un lado los sentimientos positivos que existen hacia las mujeres y que coexisten (Glick \& Fiske, 1996; Moya \& De Lemus, 2004; Moya, Expósito y Padilla, 2006). Basados en dicho fundamento se definen los dos componentes que conforman el sexismo ambivalente: el sexismo hostil (SH) el cual es concebido tradicionalmente como una actitud negativa hacia las mujeres y el sexismo benévolo (SB), definido como un conjunto de actitudes interrelacionadas hacia las mujeres que son sexistas en cuanto a que las considera de forma estereotipada y limitadas a ciertos roles, pero que tiene un tono afectivo positivo (para el perceptor) y tiende a suscitar en éste conductas típicamente categorizadas como prosociales o de búsqueda de intimidad. De acuerdo con Rodríguez, Lameiras, Carrera y Faílde (2009) los hombres sexistas evitan conflictos entre sus actitudes positivas y negativas hacia las mujeres, clasificando a las mujeres en subgrupos, uno bueno y uno malo. De esa forma los hombres realizan una categorización de las mujeres de acuerdo a la ganancia obtenida de ellas.

En este sentido Glick y Fiske (1996) diferenciaron a priori tres dimensiones que conforman tanto el sexismo hostil como el sexismo benévolo. La primera tiene que ver con la distribución del poder y se denomina paternalismo la segunda dimensión se refiere a la diferenciación de género, ya sea competitiva o complementaria y la tercera dimensión concierne a la sexualidad, en la cual las mujeres carecen de sexualidad o tienen una poderosa sexualidad que las hace peligrosas para los hombres, y por otro lado, las relaciones de pareja heterosexuales que son esenciales para alcanzar la verdadera felicidad.

Se han encontrado pocos estudios que muestren la relación existente entre el machismo o sexismo y la edad. El estudio presentado por Lameiras-Fernández y Rodríguez-Castro (2002) es un buen ejemplo. Dicho estudio se realizó con población adolescente y se encontró un mayor nivel de sexismo benévolo entre los mas jóvenes (tanto hombres como mujeres), mismo que iba en disminución a medida que aumentaba la edad. Moya, Expósito, Rodríguez-Bailon, Glick y Páez (2002) presentaron los resultados de su investigación en donde se realizó un análisis comparativo del sexismo ambivalente en España y Latinoamérica, los resultados muestran diferentes niveles de sexismo en función de la edad (a mayor edad, mayor sexismo, tanto hostil como benévolo), del grado de religiosidad (a 
mayor religiosidad, mayor sexismo, sobre todo sexismo benévolo) y del nivel educativo (a mayor educación, menor nivel de sexismo). Un tercer estudio presentado por Glick, Lameiras-Fernández y Rodríguez-Castro (2002) muestra que la educación se asoció con menor nivel de actitud sexista (benévola y hostil), y que la práctica de la religión católica se asoció a actitudes sexistas benévolas.

Cruz, Alonso y Correa (2005) en su estudio de validación del Inventario de Sexismo Ambivalente (ASI) de Glick y Fiske (1996) en México encontraron una correlación negativa entre el nivel de estudios y el sexismo ambivalente tanto en hombres como en mujeres, lo que nos indica que para estas últimas a mayor nivel de estudio decrece la dependencia que se pueda tener hacia los hombres y como resultado se tiene menor tolerancia al sexismo, Lameiras-Fernández y Rodríguez-Castro (2003) encontraron los mismos resultados de correlación negativa entre el nivel de estudios y el sexismo en su evaluación del sexismo ambivalente en estudiantes gallegos/as. De Lemus, Castillo, Moya, Padilla y Ryan (2008) en su adaptación del ASI para adolescentes encontraron que el sexismo se basa en el mantenimiento del poder y de una identidad distintiva por parte de los hombres respecto a las mujeres, de forma tal que en cuestión de sexualidad, es de esperar que los hombres obtengan puntuaciones altas en sexismo hostil debido a sus deseos ambivalentes de intimidad y dominación sexual.

La búsqueda de información nos llevó a encontrar pocos estudios que investiguen de manera directa la relación existente entre el machismo o sexismo y la salud sexual. Pleck, Sonenstein y Ku (1993) encontraron que los adolescentes que siguen una actitud masculina tradicional reportan una mayor cantidad de parejas sexuales, un menor uso consistente del condón, actitudes relacionadas con un menor uso del condón, menor creencia de responsabilidad del hombre en relación a la prevención del embarazo y, en resumen, encontraron que la ideología de la masculinidad tradicional está asociada con relaciones heterosexuales íntimas limitadas, incremento en los embarazos no deseados, así como la transmisión de infecciones de transmisión sexual (ITS) incluido el VIH (Virus de Inmunodeficiencia Humana).

La conducta sexual del hombre machista es un factor desencadenante de riesgos a la salud de la mujer. La sexualidad del machista está conformada por conductas irresponsables, irrespetuosas y egoístas, que colocan a la mujer en una situación de riesgo. Sin importar cuanto esté dispuesta la mujer a cuidarse y prevenir embarazos o infecciones de transmisión sexual o VIH/SIDA mediante la utilización del condón, la reacción del hombre machista será de cuestionarle alguna infidelidad de parte de ella.

En algunos países de Latinoamérica de acuerdo a la cultura, el hombre machista puede justificar el tener múltiples parejas sexuales y manifestar que es una necesidad, un mayor número de compañeras sexuales puede significar ser "mas hombre" y como consecuencia se encuentran en un mayor riesgo de contraer alguna ITS o VIH/SIDA y de infectar a su pareja formal así como a sus parejas sexuales ocasionales. En base a estas actitudes y comportamientos podemos definir el machismo sexual como creencia de superioridad del hombre que deriva en conductas sexuales que se llevan a cabo y que ponen en riesgo la salud sexual y el bienestar físico propio así como de su (s) pareja (s).

El objetivo de elaborar la Escala de Machismo Sexual (Sexismo) (EMS-Sexismo-12) que aquí se presenta, es el de evaluar conductas, actitudes y creencias machistas desde la perspectiva sexual y de esa manera conocer los niveles de riesgo que se manifiestan al ejercer o tolerar el machismo en una situación que puede tornarse de riesgo a la vida como lo es la sexualidad. Se considera elemental realizar esta escala con el fin de detectar situaciones de riesgo y de esta manera evitar la propagación de infecciones de transmisión sexual así como un incremento en los casos de VIH/SIDA.

Es así, que los objetivos del estudio fueron presentar los análisis de validez y confiabilidad del EMS-Sexismo-12, comparar el nivel de machismo entre hombres y mujeres y valorar la correlación con la edad de los sujetos. 


\section{Método}

\section{Muestra}

Participaron en el estudio 79 sujetos, de los cuales 43 eran hombres y 36 eran mujeres. El grupo de los hombres presentó una media de edad de 30.65 años (D.E. $=18.80$ ); el grupo de las mujeres mostró una media de edad de 26.42 (D.E.=12.19).

Se realizó un muestreo por conveniencia. Los sujetos fueron invitados a participar de manera voluntaria, la aplicación del cuestionario tuvo lugar en áreas públicas concurridas de la ciudad de Monterrey, N.L., México

\section{Instrumento de evaluación}

El instrumento fue elaborado por las autoras de este artículo en base a la definición de machismo mencionada por Castañeda (2002, 2007). Inicialmente, la Escala de Machismo Sexual (Sexismo), quedó conformada por 24 ítems. Los ítems tienen una puntuación de 1 (totalmente en desacuerdo) a 5 (totalmente de acuerdo). La puntuación mínima es de 24 y la máxima es de 120. Se realizó una prueba piloto y los datos fueron analizados mediante análisis de consistencia interna, así mismo se recodificaron 14 ítems para la evaluación. Al realizar los análisis del instrumento se encontraron resultados inadecuados en 12 ítems; se realizaron adecuaciones a la escala eliminando los ítems inadecuados y se evaluaron los ítems restantes de la escala mediante el análisis de consistencia interna y análisis factorial exploratorio y confirmatorio, dando como resultado 12 ítems con una puntuación mínima de 12 y máxima de 60.

\section{Procedimiento de análisis estadístico}

Se inició con el análisis de consistencia interna y la evaluación de la correlación inter-ítem, posteriormente se llevó a cabo un análisis factorial exploratorio (AFE), con el método de componentes principales para evaluar el número de factores recomendados por el gráfico de sedimentación y la regla K1. Se probaron soluciones con diferente número de factores y rotación varimax para maximizar la independencia entre factores. Con base en los resultados se conservó la solución unifactorial con 12 ítems. Una vez reducida la escala se estimó nuevamente la consistencia interna. El cuestionario reducido a 12 ítems fue sometido a AFC para obtener los estadísticos de bondad de ajuste de la versión reducida del cuestionario. Para el análisis factorial confirmatorio se utilizó el programa AMOS y se trabajó con el método de máxima verosimilitud.

\section{Resultados}

Se evaluó la escala completa, constituida por 24 ítems mediante el alfa de Cronbach, los resultados muestran un alfa de .83, este valor puede ser considerado adecuado, sin embargo, se procedió a analizar la correlación inter-ítem y de esta manera tomar decisiones sobre los ítems a eliminar para reducir la escala. La tabla 1 muestra los resultados.

Tabla 1. Correlación inter-ítem y consistencia interna al eliminar cada ítem

\begin{tabular}{lcc}
\hline & $\begin{array}{c}\text { Correlación } \\
\text { ínter-item }\end{array}$ & $\begin{array}{c}\text { Alfa si el ítem es } \\
\text { eliminado }\end{array}$ \\
\hline 1. Que solamente el hombre tenga sexo antes del matrimonio. & .568 & .821 \\
2. Que un hombre tenga hijos fuera del matrimonio. & .449 & .827 \\
3. Que una mujer engañe a su pareja. & -.101 & .845
\end{tabular}


4. Que solamente el hombre tenga experiencia sexual.

pareja.

7. Que un hombre casado o con pareja estable tenga relaciones sexuales con prostitutas.

8. Que una mujer tenga experiencia sexual.

9. Que sea el hombre quien se encargue de tener condones para la relación sexual.

10. Una mujer debe aceptar las infidelidades de su pareja.

11. Que la mujer pida a su pareja tener relaciones sexuales.

12. Que tu pareja haya tenido experiencias sexuales antes de estar contigo.

13. Que la mujer se niegue a tener relaciones sexuales cuando su pareja se lo pide.

14. Que la mujer se masturbe.

15. Que una mujer tenga su primera relación sexual con una persona que no es su pareja.

16. El hombre necesita tener varias parejas sexuales.

17. Que la mujer asista a un table dance.

18. Sin importar la situación o el estado de ánimo, la mujer debe tener relaciones sexuales cuando su pareja quiera tenerlas.

19. Que un hombre tenga varias parejas sexuales al mismo tiempo

20. Que un hombre se masturbe.

21. Que un hombre asista a un table dance.

22. Que sea la mujer quien se encargue de cuidarse para la relación sexual o para no tener hijos.

23. El hombre debe iniciar su vida sexual en la adolescencia.

24. El hombre debe hacer que su hijo hombre inicie su vida sexual (tenga su primera relación sexual).
.561

.247

.822

Se puede observar que los ítems 3 y 20 correlacionan negativamente, y las correlaciones de los ítems 5, 6, 8, 9, 11, 14, 15, 17 y 21 son débiles. Antes de reducir la escala, se procedió a analizar la escala con el Análisis Factorial Exploratorio (con los ítems sin recodificar). El gráfico de sedimentación sugiere al menos 3 factores. El porcentaje de varianza explicada con 1 factor fue de $28.45 \%$, con 2 factores de $44.17 \%$ y con 3 factores de 52.36\%. Sin embargo, la regla K1 sugiere 6 factores.

Posteriormente se procedió a realizar un segundo Análisis Factorial Exploratorio con tres factores. Al considerar cargas factoriales superiores a .40, el factor uno, conformado por los ítems 1 , $2,4,6,7,10,16,18,19,22,23$, y 24, agrupa las preguntas relacionadas directamente al rol activo del hombre, así como la libertad de ejercer y practicar su sexualidad y del rol pasivo de la mujer en cuanto a la decisión de tener relaciones sexuales o mostrar inconformidad por la infidelidad de parte del hombre. El factor dos está integrado por los ítems 3, 5, 8, 12, 13 y 15, dicho factor agrupa las 
preguntas relacionadas a las decisiones de la mujer en cuanto a su vida sexual, así como del papel activo de la mujer en la sexualidad. El factor 3 se haya conformado por los ítems 14, 17, 20 y 21, los cuales están enfocados a la opinión de la mujer en cuanto a la sexualidad, las actividades de masturbación tanto del hombre como de la mujer y el inicio de la vida sexual de la mujer. Los ítems 9 y 11 no alcanzan carga factorial superior a .40 en ninguno de estos 3 factores. Esta solución resulta interpretable, de acuerdo al diseño original de la escala.

Una vez analizados los ítems agrupados por factores se decide conservar exclusivamente el primer factor, ya que esos ítems son considerados representativos de la definición de machismo tomada para la presente investigación. Se realizó de nuevo el análisis de la escala compuesta ahora por 12 ítems mediante el alfa de Cronbach obteniendo ahora un valor adecuado de alfa de .91 y correlaciones fuertes entre todos los ítems (Tabla 2).

Tabla 2. Correlación inter-ítem de la versión reducida de la Escala de Machismo Sexual (Sexismo)

\begin{tabular}{|c|c|}
\hline Ítem & $\begin{array}{c}\text { Correlación } \\
\text { inter-ítem }\end{array}$ \\
\hline 1. Que solamente el hombre tenga sexo antes del matrimonio. & .618 \\
\hline 2. Que un hombre tenga hijos fuera del matrimonio. & .580 \\
\hline 3. Que solamente el hombre tenga experiencia sexual. & .618 \\
\hline $\begin{array}{l}\text { 4. Que un hombre tenga su primera relación sexual con una persona que no } \\
\text { es su pareja. }\end{array}$ & .489 \\
\hline $\begin{array}{l}\text { 5. Que un hombre casado o con pareja estable tenga relaciones sexuales } \\
\text { con prostitutas. }\end{array}$ & 620 \\
\hline 6. Una mujer debe aceptar las infidelidades de su pareja. & .665 \\
\hline 7. El hombre necesita tener varias parejas sexuales. & .774 \\
\hline $\begin{array}{l}\text { 8. Sin importar la situación o el estado de ánimo, la mujer debe tener rela- } \\
\text { ciones sexuales cuando su pareja quiera tenerlas. }\end{array}$ & .745 \\
\hline 9. Que un hombre tenga varias parejas sexuales al mismo tiempo. & .762 \\
\hline $\begin{array}{l}\text { 10. Que sea la mujer quien se encargue de cuidarse para la relación sexual } \\
\text { o para no tener hijos. }\end{array}$ & .559 \\
\hline 11. El hombre debe iniciar su vida sexual en la adolescencia. & .597 \\
\hline $\begin{array}{l}\text { 12. El hombre debe hacer que su hijo hombre inicie su vida sexual (tenga } \\
\text { su primera relación sexual). }\end{array}$ & .689 \\
\hline
\end{tabular}

Se valoró nuevamente la estructura factorial de los 12 ítems con Análisis Factorial Exploratorio obteniendo un porcentaje de varianza explicada con un solo factor de 50.82. Así mismo se muestran pesos factoriales adecuados para los 12 ítems y un factor sugerido por el gráfico de sedimentación y la regla $\mathrm{K} 1$. 
Con base en el AFE se estimó un modelo de ecuaciones estructurales para realizar el Análisis factorial confirmatorio (AFC); incluyendo los 12 ítems en un solo factor, los resultados del AFE y del análisis de consistencia interna sugieren que este modelo sería el adecuado.

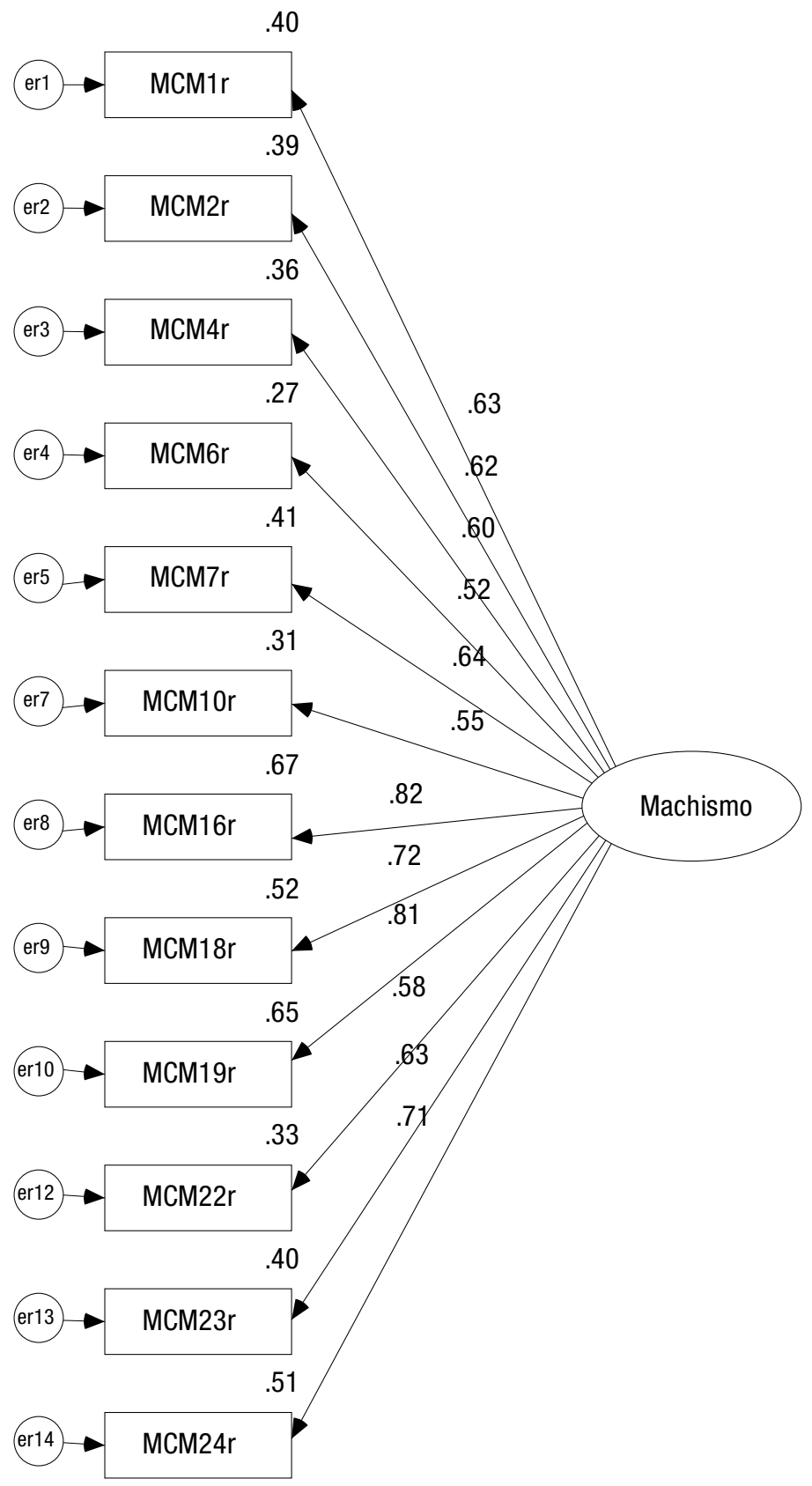

En este modelo estructural, todos los parámetros resultaron significativos. El porcentaje de varianza explicada para machismo en el AFC fue de $98.1 \%$, asimismo, el modelo presenta estadísti$\cos$ de bondad de ajuste aceptables $\left(\chi^{2}(54)=78.380, \mathrm{p}=.017 ; \chi 2 / \mathrm{gl}=1.451 ; \mathrm{GFI}=.868 ; \mathrm{AGFI}=.810\right.$; $\mathrm{CFI}=.936 ; \mathrm{TLI}=.922 ; \mathrm{IFI}=.938 ; \mathrm{RMSEA}=.076)$. 
Las medidas de tendencia central para la versión final de la escala, con 12 ítems se presentan en la tabla 3, con la muestra completa y separando de acuerdo al sexo. La variable no se ajusta a una distribución normal $\left(\mathrm{Z}_{\mathrm{K}-\mathrm{s}}=0.147\right.$; $\left.\mathrm{p}=.001\right)$, por lo que al comparar hombres y mujeres se utilizó la prueba $U$ de Mann-Whitney, encontrando una diferencia significativa $(Z=-3.095 ; p=.002)$, siendo los hombres quienes presentan puntajes más altos. Por último, no se encontró correlación significativa con la edad $\left(\mathrm{r}_{\mathrm{s}}=-.075 \mathrm{p}=.511\right)$

Tabla 3. Medidas de tendencia central y desviación estándar de la Escala de Machismo Sexual (Sexismo)

\begin{tabular}{lccc}
\hline & Mediana & Media & Desviación estándar \\
\hline Muestra completa & 22.0 & 25.7 & 10.23 \\
Hombres $\mathrm{n}=43$ & 26.0 & 28.8 & 11.4 \\
Mujeres $\mathrm{n}=36$ & 21.0 & 22.1 & 7.2 \\
\hline
\end{tabular}

\section{Discusión}

La elaboración de la presente escala surge debido a la falta de instrumentos en el país que nos ayuden en la evaluación de aspectos cognitivos (machismo/sexismo) que pueden tener una repercusión en aspectos físicos (salud sexual/riesgo de ITS, VIH/SIDA y embarazos no deseados). El propósito de elaborar la Escala de Machismo Sexual (Sexismo) (EMS-Sexismo-12) consistió en contar con un instrumento breve, válido y confiable para evaluar conductas, actitudes y creencias machistas desde la perspectiva sexual, sin embargo, los análisis realizados nos llevaron a una escala unifactorial que evalúa las creencias machistas que pueden tener hombres y mujeres en relación a la sexualidad del propio sexo y del sexo opuesto. La escala fue elaborada considerando su aplicación en nuestro país, así como otros países de habla hispana donde el machismo/sexismo sea parte de su cultura, ya que las preguntas fueron realizadas con un lenguaje adecuado y genérico en cuestión de edad, sexo y preferencia sexual; se aplicó una prueba piloto y se realizaron modificaciones logrando así un porcentaje bajo de preguntas sin contestar.

$\mathrm{Al}$ analizar los resultados obtenidos con el AFE se encontró que las características principales del machismo en México siguen bajo la definición clásica del sexismo o sexismo hostil (Glick \& Fiske, 1996). Como se puede ver en los ítems que conforman el factor 1 y que conforman finalmente la versión final de la escala EMS-Sexismo-12, el rol activo de la mujer en la toma de decisiones y participación en la sexualidad es más bien considerado como una contraparte del rol del hombre en la sexualidad, en donde sólo uno de los miembros de la pareja puede tomar las decisiones y controlar la relación.

Se observan diferencias claras entre las opiniones de hombres y mujeres. Esto puede relacionarse directamente con la teoría del sexismo ambivalente de Glick y Fiske (1996) donde señalan la postura machista del papel de las mujeres en cuanto a la sexualidad, proponen que las mujeres carecen de sexualidad, o bien que la sexualidad que ejercen representa una amenaza para el hombre, por lo que se le otorga un nivel de inferioridad.

El concepto de machismo engloba posturas de tipo cultural, laboral, familiar, entre otros; sin embargo la aportación específica de este instrumento, consiste en la medición del grado de machismo de hombres y mujeres, en lo referente a las cuestiones sexuales. Este abordaje permite encaminar el concepto de machismo a investigaciones de salud sexual con mayor precisión que los instrumentos que miden sexismo desde la totalidad del concepto. La escala puede tener alcance en investigacio- 
nes de género, violencia y salud sexual, así mismo puede ser útil en la evaluación de adolescentes y adultos así como a personas de cualquier género y preferencia sexual, ya que mide las creencias personales de cada individuo en relación al machismo.

Se logra tener una escala breve con validez y confiabilidad para medir el machismo sexual, a pesar que el cuestionario fue aplicado en la ciudad de Monterrey, N.L. y sólo se evaluó a esta comunidad se puede afirmar que su lenguaje es adecuado para ser aplicado en otras ciudades del resto del país incluso en otros países de Latinoamérica.

\section{Referencias}

Castañeda, M. (2002). El machismo invisible. Mexico: Grijalbo.

Castañeda, M. (2007). El machismo invisible regresa. Mexico: Santillana.

Cruz, C.; Alonso, V. \& Correa, F. (2005). Perfiles de sexismo en la ciudad de México: Validación del cuestionario de medición del sexismo ambivalente. Enseñanza e investigación de psicología. 10, (2), 381-395.

De Lemus, S.; Castillo, M.; Moya, M.; Padilla, J.L. \& Ryan, E. (2008). Elaboración y validación del Inventario de Sexismo Ambivalente para Adolescentes. International Journal of Clinical and Health Psychology, 8, (2), 637-562.

Glick, P. \& Fiske, T. (1996). The Ambivalent Sexism Inventory: Differentiating Hostile and Benevolent Sexism. Journal of Personality and Social Psychology, 70, (3), 491-512.

Lameiras-Fernández, M. \& Rodríguez-Castro, Y. (2002). Evaluación del sexismo moderno enadolescentes. Revista de Psicología Social, 17, 119-127

Lameiras-Fernández, M. \& Rodríguez-Castro, Y. (2003). Evaluación del sexismo ambivalente en estudiantes gallegos/as. ACCIÓN PSICOLÓGICA, 2, (2), 131-136.

Moya, M. \& Expósito, F. (2001). Nuevas formas, viejos intereses: neosexismo en varones españoles. Psicothema, 13, (4), 643-649.

Moya, M. \& De Lemus, S. (2004). Superando barreras: Creencias y aspectos motivacionales relacionados con el ascenso de las mujeres a puestos de poder. Revista de Psicología General y Aplicada, 57, (2), 225-242-

Moya, M.; Expósito, F. \& Padilla, J.L. (2006). Revisión de las propiedades psicométricas de las versiones larga y reducida de la escala sobre ideología de género. International Journal of Clinical and Health Psychology, 6, (3), 709-727.

Pleck, J.H.; Sonenstein, F.L. \& Ku, L.C. (1993). Masculinity ideology: Its impact on adolescent males' heterosexual relashionships. Journal of Social Issues, 49, (3), 11-29.

Rodríguez, Y.; Lameiras, M.; Carrera, M.V. \& Faílde, J.M. (2009). Aproximación conceptual al sexismo ambivalente: Estado de la cuestión. SUMMA Psicológica UST, 6, (2), 131-142.

Villegas, M. (2004). El destino del macho. Mexico: Oficio. 


\section{ANEXO}

\section{Escala de Machismo Sexual (EMS-Sexismo-12)}

Expresa en tu opinión tu grado de acuerdo o desacuerdo con las siguientes frases. Por favor responde honestamente utilizando estas opciones: (1) Totalmente en desacuerdo; (2) En desacuerdo; (3) Sin opinión; (4) De acuerdo; (5) Totalmente de acuerdo

\begin{tabular}{|c|c|c|c|c|c|}
\hline & $\begin{array}{l}\text { Totalmente en } \\
\text { desacuerdo }\end{array}$ & En desacuerdo & Sin opinión & De acuerdo & $\begin{array}{l}\text { Totalmente de } \\
\text { acuerdo }\end{array}$ \\
\hline $\begin{array}{l}\text { 1. Que solamente el hombre tenga } \\
\text { sexo antes del matrimonio }\end{array}$ & 1 & 2 & 3 & 4 & 5 \\
\hline $\begin{array}{l}\text { 2. Que un hombre tenga hijos fuera } \\
\text { del matrimonio }\end{array}$ & 1 & 2 & 3 & 4 & 5 \\
\hline $\begin{array}{l}\text { 3. Que solamente el hombre tenga } \\
\text { experiencia sexual }\end{array}$ & 1 & 2 & 3 & 4 & 5 \\
\hline $\begin{array}{l}\text { 4. Que un hombre tenga su primera } \\
\text { relación sexual con una persona que } \\
\text { no es su pareja }\end{array}$ & 1 & 2 & 3 & 4 & 5 \\
\hline $\begin{array}{l}\text { 5. Que un hombre casado o con } \\
\text { pareja estable tenga relaciones } \\
\text { sexuales con prostitutas }\end{array}$ & 1 & 2 & 3 & 4 & 5 \\
\hline $\begin{array}{l}\text { 6. Una mujer debe aceptar las } \\
\text { infidelidades de su pareja }\end{array}$ & 1 & 2 & 3 & 4 & 5 \\
\hline $\begin{array}{l}\text { 7. El hombre necesita tener varias } \\
\text { parejas sexuales }\end{array}$ & 1 & 2 & 3 & 4 & 5 \\
\hline $\begin{array}{l}\text { 8. Sin importar la situación o el } \\
\text { estado de ánimo, la mujer debe tener } \\
\text { relaciones sexuales cuando su pareja } \\
\text { quiera tenerlas }\end{array}$ & 1 & 2 & 3 & 4 & 5 \\
\hline $\begin{array}{l}\text { 9. Que un hombre tenga varias } \\
\text { parejas sexuales al mismo tiempo }\end{array}$ & 1 & 2 & 3 & 4 & 5 \\
\hline $\begin{array}{l}\text { 10. Que sea la mujer quien se } \\
\text { encargue de cuidarse para la relación } \\
\text { sexual }\end{array}$ & 1 & 2 & 3 & 4 & 5 \\
\hline $\begin{array}{l}\text { 11. El hombre debe inicia su vida } \\
\text { sexual en la adolescencia }\end{array}$ & 1 & 2 & 3 & 4 & 5 \\
\hline $\begin{array}{l}\text { 12. El hombre debe hacer que su hijo } \\
\text { hombre inicie su vida sexual }\end{array}$ & 1 & 2 & 3 & 4 & 5 \\
\hline
\end{tabular}

